\title{
AN ON-BOARD FIELD AREA METER FOR AGRICULTURAL MACHINERY
}

\author{
Yuan-Yong Hsu \\ Department of Vehicle Engineering, National Formosa University, Huwei, Yunlin 63201, Taiwan, R.O.C, \\ yonger@nfu.edu.tw \\ Kuo-Ching Chiou \\ Department of Vehicle Engineering, National Formosa University, Huwei, Yunlin 63201, Taiwan, R.O.C \\ Cheng-Ta Chung \\ Department of Vehicle Engineering, National Formosa University, Huwei, Yunlin 63201, Taiwan, R.O.C
}

Tsung-Hua Hsu

Department of Mechanical and Electro-Mechanical Engineering, National Formosa University, Huwei, Yunlin 63201, Taiwan, R.O.C

Follow this and additional works at: https://jmstt.ntou.edu.tw/journal

Part of the Engineering Commons

\section{Recommended Citation}

Hsu, Yuan-Yong; Chiou, Kuo-Ching; Chung, Cheng-Ta; and Hsu, Tsung-Hua (2010) "AN ON-BOARD FIELD AREA METER FOR AGRICULTURAL MACHINERY," Journal of Marine Science and Technology. Vol. 18: Iss. 4, Article 5.

DOI: 10.51400/2709-6998.1903

Available at: https://jmstt.ntou.edu.tw/journal/vol18/iss4/5

This Research Article is brought to you for free and open access by Journal of Marine Science and Technology. It has been accepted for inclusion in Journal of Marine Science and Technology by an authorized editor of Journal of Marine Science and Technology. 


\section{AN ON-BOARD FIELD AREA METER FOR AGRICULTURAL MACHINERY}

\section{Acknowledgements}

The authors are indebted to Lee-Guan Factory and YuanChen Co., Ltd. for providing a corn harvester and a peanut gathering machine for testing. Furthermore, the authors would like to thank for the financial support from Ministry of Economic Affairs (CIUP project NO. 92U0082) in Taiwan and the assistance of professor Lee Bean-Yin 


\title{
AN ON-BOARD FIELD AREA METER FOR AGRICULTURAL MACHINERY
}

\author{
Yuan-Yong Hsu*, Kuo-Ching Chiou*, Cheng-Ta Chung*, and Tsung-Hua Hsu**
}

Key words: agricultural machinery, agriculture automation.

\begin{abstract}
In this work, an on-board field area meter with simple structure and low cost is developed on agricultural machinery in order to measure the cultivated area. It consists of a simple compass for position measurement and a proximity switch for distance measurement. Furthermore, an 8051 series microprocessor is employed to perform the calculations required for calibration and contour integral. The device is designed to be operated in either manual or automatic mode. Field tests carried out on a peanut gathering machine and a corn harvester show that the errors of the measured area in both operation modes are less than $2.5 \%$. Hence, it is highly expected that this device can offer great contribution to agricultural automation.
\end{abstract}

\section{INTRODUCTION}

Agricultural machinery requires position data for application as precision farming [1] and a network service [5]. In addition, farming workers, who operate agricultural machinery such as corn harvesters and peanut gathering machinery, etc., usually need to measure the field area they have cultivated in order to calculate the corresponding pay. However, there always exists dispute on the amount of payment between workers and employers due to the lack of accurate cultivated area. Therefore, it is essential for the farming business to develop a simple device which can be used to measure the cultivated field area in a very accurate and efficient manner.

A common method to calculate the area of an irregular closed contour is to allocate several points on this contour using an absolute or relative coordinate. Then, a vector method is used to calculate the areas of the polygons generated by these points. Finally, an approximate value of area for the contour is obtained by summing up these areas. Generally, the

Paper submitted 04/23/09; revised 06/10/09; accepted 07/21/09. Author for correspondence: Yuan-Yong Hsu (e-mail: yonger@nfu.edu.tw).

*Department of Vehicle Engineering, National Formosa University, Huwei, Yunlin 63201, Taiwan, R.O.C.

**Department of Mechanical and Electro-Mechanical Engineering, National Formosa University, Huwei, Yunlin 63201, Taiwan, R.O.C. more points on the contour are selected, the higher accuracy of the calculation can be attained. The common shapes of fields are rectangle or polygon. Therefore, a sufficiently accurate result can be gained by only choosing the points of turn. An absolute position for a selected point can be got by directly receiving the signal emitted by global positioning system (GPS). However, its position error is beyond $3 \mathrm{~m}$. The error of calculated area may be higher than $50 \%$ for fields with area less than one hectare. If differential global positioning system (DGPS) using signal stations on the ground for calibration is applied instead [3], the errors can be significantly reduced to about $1 \mathrm{~m}$. Unfortunately, these stations are usually located in urban area and too far away from fields in a countryside to be utilized for DGPS. Therefore, a more economic and feasible way is to obtain their positions based on a relative coordinate system using an azimuth sensor and a distance sensor. For this purpose, a novel on-board field area meter (OBFAM) with an azimuth sensor and a distance sensor is proposed to provide measured area data for agricultural machinery.

\section{SYSTEM STRUCTURE}

To fulfill the basic requirements of easy installation, low manufacturing cost, high reliability, high accuracy, and satisfactory performance on a tough environment etc., a fluxgate magnetic compass $[2,4]$ available on the market is adopted as the position sensor. In addition, a distance sensor mounted on a wheel shaft is designed by ourselves. A single-chip W78E516 microprocessor is utilized to perform position calibration and integral calculation required for estimating distance as well as area. The schematic layout of OBFAM is illustrated in Fig. 1. In the following section, the major components of OBFAM will be briefly discussed.

\section{Azimuth Sensor}

An azimuth sensor as shown in Fig. 2, which is a commercial product of Topteam Technology CO., LTD. and costs only $\$ 15$, is employed to measure the azimuth angle by detecting the magnet of the earth.

The basic structure diagram of the azimuth sensor is shown Fig. 3. An exciting coil is wound around a circular strongmagnetic Fe-Ni core. Coil $\mathrm{X}$ is wound across the outer surfaces of the upper and lower rim of the core and is connected 


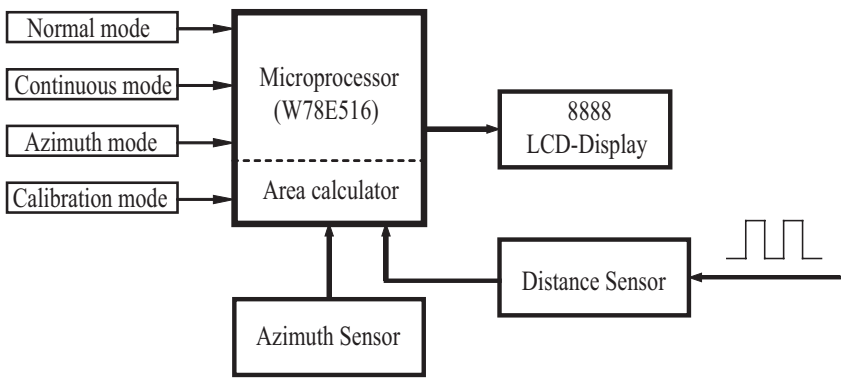

Fig. 1. Schematic layout of OBFAM.

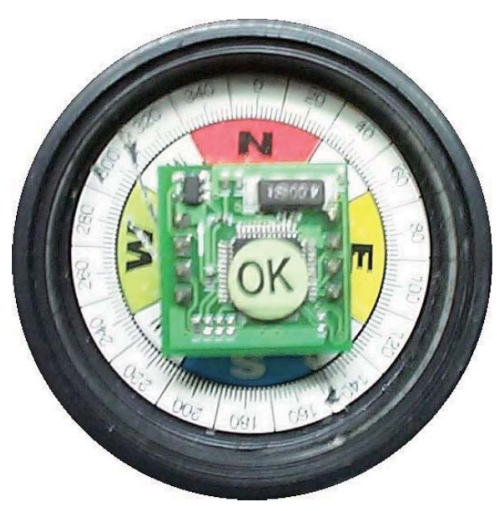

(a) Front view of the azimuth sensor

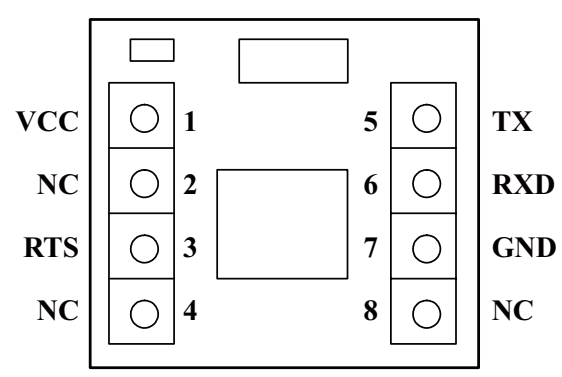

(b) Layout of input/output interface

Fig. 2. Illustration of the azimuth sensor.

to a potential meter for detecting the voltage induced by a horizontal magnetic field passing through the loop of Coil X; similarly, Coil Y is wound across the outer surface of the right and left rim of the core and is connected to a potential meter for detecting the voltage induced by a vertical magnetic field passing through the loop of Coil Y. Supplied power from an alternating voltage source, the exciting coil creates an alternating magnetic field within the circular core, e.g. the magnetic fluxes $\varphi_{1}$ and $\varphi_{2}$ for positions $S_{1}$ and $S_{2}$ in Fig. 3, respectively. It is required that the magnetic field should not be saturated. When there is no external magnetic field, the intensities of the magnetic fluxes $\varphi_{1}$ and $\varphi_{2}$ at positions $S_{1}$ and $S_{2}$ are equal and their directions are opposite. Thus, the net magnetic fluxes through Coil $\mathrm{X}$ and Coil $\mathrm{Y}$ are both zero and the induced voltages $V_{x}$ and $V_{y}$ are also zero $(V=-N \cdot d \varphi / d t, N$ : turn number of coil).

When the magnetic field of the earth is present, it will

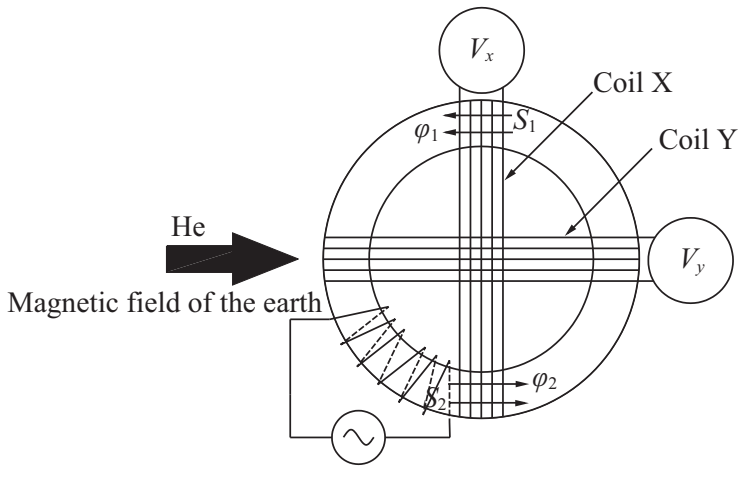

Fig. 3. Basic structure of the azimuth sensor.

change the net magnetic fluxes of Coil $\mathrm{X}$ and Coil $\mathrm{Y}$ as well as the induced voltages $V_{x}$ and $V_{y}$ respectively indicated as

$$
\begin{aligned}
& V_{x}=K B \cos \theta \\
& V_{y}=K B \sin \theta
\end{aligned}
$$

where $K$ is the coherent coefficient of Coil X or Coil Y, $B$ is the horizontal component of the magnetic intensity of the earth, and $\theta$ is the azimuth angle of the magnetic field of the earth relative to the azimuth sensor. When the sensor is placed in a vehicle, the instantaneous azimuth angle of the moving vehicle can be obtained by

$$
\theta=\tan ^{-1}\left(V_{y} / V_{x}\right)
$$

which is derived from (1) and (2). In addition, by taking square of (1) and (2) and summing them up, we get

$$
V_{\mathrm{x}}^{2}+V_{y}^{2}=(K B)^{2}
$$

that indicates a circular trajectory with radius of $K B$ on the $x-y$ coordinate. This circle is called the azimuth principle circle.

As this type of sensors detects the magnetic field of the earth for measuring the azimuth angle, an error will be generated when the sensors are subject to other external magnetic fields. As shown in Fig. 4, when an external magnetic field with a direction angle of $\alpha$ and magnitude $G$ is present, the voltage state will deviate from point $A$ to point $B$. The resulting error of the azimuth angle $\varphi$ can be represented as

$$
\varphi=\tan ^{-1} \frac{V_{y}}{V_{x}}-\tan ^{-1} \frac{V_{y}-K G \sin \alpha}{V_{x}-K G \cos \alpha}
$$

Generally, there are two types of external magnetic fields that will cause an error in the application of agricultural machinery. One is the residual magnetic field in the vehicle body. Its intensity is normally much smaller than that of the magnetic field of the earth. This type of error can be corrected 


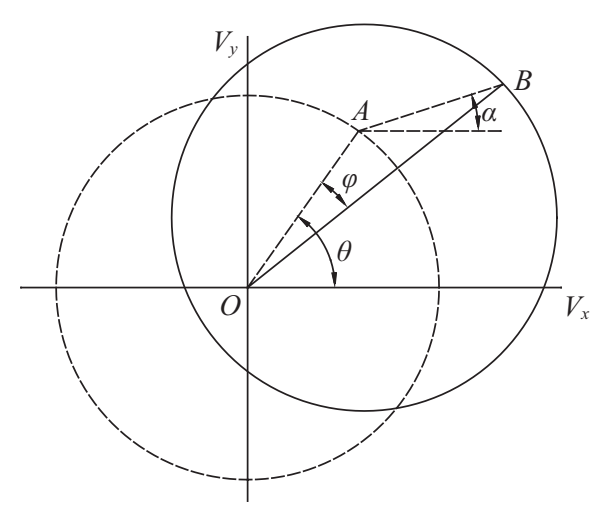

Fig. 4. Azimuth principle circle.

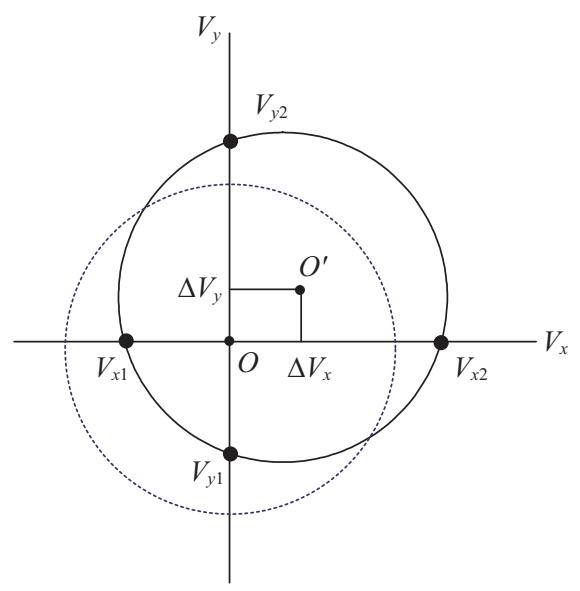

Fig. 5. Principle of the automatic calibration method.

using a so-called automatic-calibration method. The other is the magnetic field originated from some electrical power sources such as a transmission line. Its intensity is usually several times stronger than that of the magnetic field of the earth. This type of error requires a so-called cyclic calibration method. These two methods are described as follow.

\section{1) Automatic Calibration Method}

In the case of a tested vehicle with a residual magnetic field, it is rotated by $360^{\circ}$. In this case, the center of the resulting azimuth principle circle will shift away from origin $O$ along the direction of the residual magnetic field to the point $O^{\prime}$, as indicated in Fig. 5. Then, the voltage offsets respect to $V_{x}$ and $V_{y}$ from $O$ to $O^{\prime}$ are

$$
\Delta V_{x}=\frac{V_{x 1}+V_{x 2}}{2}, \quad \Delta V_{y}=\frac{V_{y 1}+V_{y 2}}{2}
$$

where $V_{x 1}, V_{x 2}, V_{y 1}$ and $V_{y 2}$ are the intercepts of the azimuth principle circle on $\mathrm{X}$ axis and $\mathrm{Y}$ axis. The required calculations to obtain $\Delta V_{x}$ and $\Delta V_{y}$ are carried out by the microprocessor.

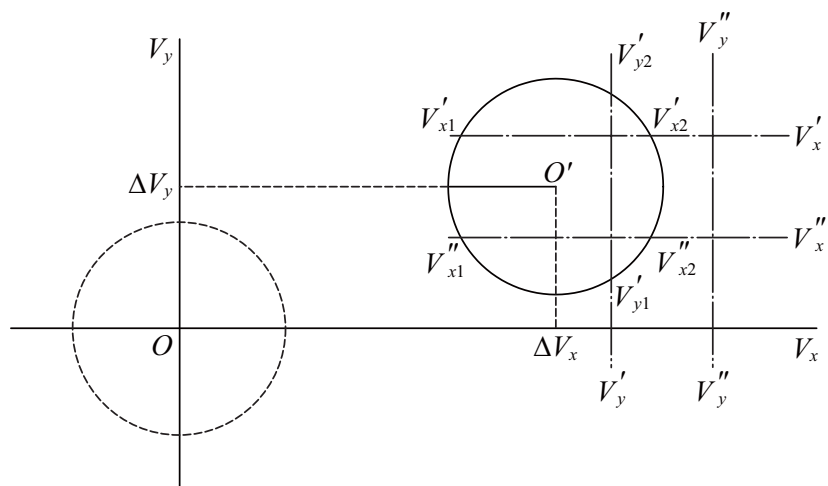

Fig. 6. Principle of the cyclic calibration method.

\section{2) Cyclic Calibration Method}

As the vehicle moves below a transmission line, the sensor will be subjected to a residual magnetic field several times stronger than that of the earth. It is required to take several sampling signals for some specific points and evaluate the voltage offsets using a method called cyclic calibration method which is described below.

As a vehicle body is subject to a strong residual magnetic field, we first acquire a set of output signal data by rotating for $360^{\circ}$ and obtain an azimuth principle circle centered at point $O^{\prime}$ indicated in Fig. 6. With the aid of a built-in calibration circuit, we locate four new coordinate axes $V_{x}^{\prime}, V_{x}^{\prime \prime}, V_{y}^{\prime}$ and $V_{y}^{\prime \prime}$ that are parallel to $\mathrm{x}$-axis or $\mathrm{y}$-axis. Then, we rotate the vehicle and generate eight output signals $V_{x 1}^{\prime}, V_{x 2}^{\prime}, V_{x 1}^{\prime \prime}, V_{x 2}^{\prime \prime}$, $V_{y 1}^{\prime}, V_{y 2}^{\prime}, V_{y 1}^{\prime \prime}$ and $V_{y 2}^{\prime \prime}$ on the circle are the intercepts of the azimuth principle circle on the four axes. Accordingly, the deviation between the circle at center $O^{\prime}$ and that at center $O$ under a strong residual magnetic field can be calculate as follow:

$$
\Delta V_{x}=\frac{V_{x 1}^{\prime}+V_{x 2}^{\prime}}{2}, \quad \Delta V_{\mathrm{y}}=\frac{V_{\mathrm{y} 1}^{\prime}+V_{\mathrm{y} 2}^{\prime}}{2}
$$

In practice, correcting the measured output signal by the deviation values $\Delta V_{x}$ and $\Delta V_{y}$ shown in (7), we can then obtain an accurate value of azimuth angle.

\section{Distance Sensor}

The distance sensor is mounted on the wheel shaft of the vehicle as shown in Fig. 7. As the wheel shaft is rotating, a square-wave signal is generated through proximity switch and related electronic circuit. By using the counter embedded in the microprocessor, the distance that the vehicle has undergone can then be calculated.

\section{Area Calculator}

To calculate the cultivated area, an area calculator is de- 


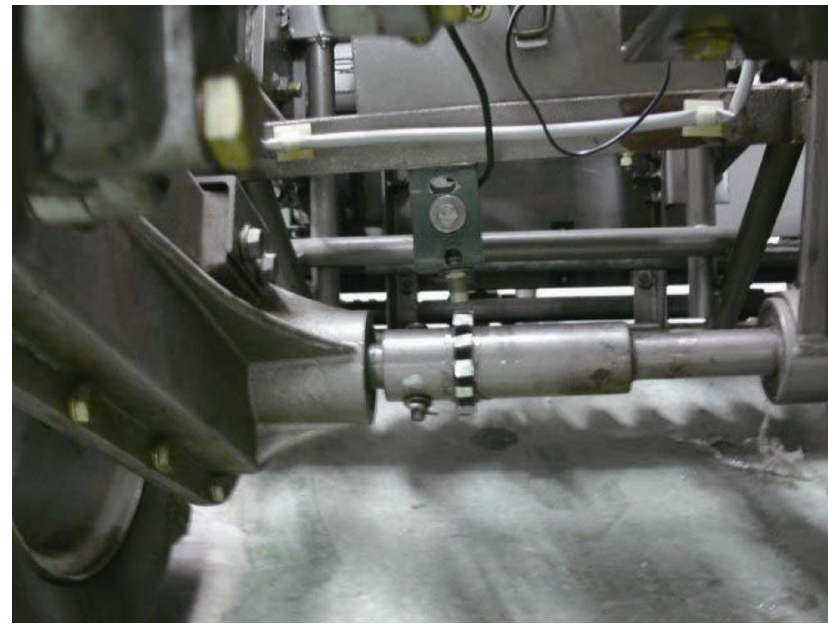

Fig. 7. View of the distance sensor.

veloped on a W78E516 microprocessor of 8051 series with on-chip $64 \mathrm{k}$ EEPROM. The developed calculator can process the signal data of azimuth and distance in a real-time mode. As the vehicle moves from a starting point along the contour of the field to be measured, the calculator calculates the positions of several selected points relative to the starting point. The selected point $i$ is represented by a two-dimensional vector $\vec{P}_{i}(x, y)$ as shown bellow:

$$
\begin{gathered}
x=\sum_{i=1}^{n} l \cdot P_{i} \cdot \cos \left(\tan ^{-1} \frac{V_{y 1}}{V_{x 1}}\right) \\
\mathrm{y}=\sum_{i=1}^{n} l \cdot P_{i} \cdot \sin \left(\tan ^{-1} \frac{V_{y 1}}{V_{x 1}}\right)
\end{gathered}
$$

where $l$ is the traveling distance of the vehicle per impulse, $P_{i}$ is the number of impulses counted as the vehicle travels from point $\vec{P}_{i-1}$ to point $\vec{P}_{i}, V_{x i}$ is induced voltage on $x$-axis direction for point $\vec{P}_{i}$, and $V_{y i}$ is induced voltage on $y$-axis direction for point $\vec{P}_{i}$. Therefore, the total area can be obtained by summing up the area of the triangle formed by the selected points, that is,

$$
\text { Area }=\frac{1}{2} \sum_{i=1}^{n-2}\left|\vec{P}_{i} \times \vec{P}_{i+1}\right|
$$

Where $n$ is the number of the selected points and $\vec{P}_{i}$ is the two-dimensional position vector $\left(x_{i}, y_{i}\right)$ representing the selected point $i$.

\section{DESIGNING AN INTEGRATED PROTOTYPE}

A commercial software called PROTEL 98 is utilized to

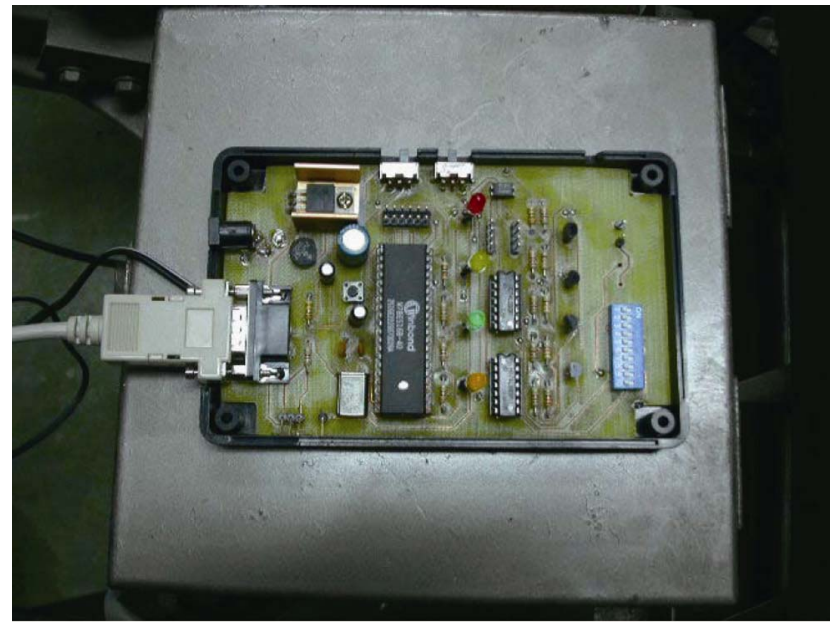

Fig. 8. View of the OBFAM prototype.

design the integrated layout of electronic circuit of the OBFAM. A desirable feature of this software is that the major procedures for designing an electronic circuit including layout drawing, circuit board design, and circuit performance simulation are integrated so that it is a powerful tool for circuit design and analysis.

The developed prototype of the OBFAM is shown in Fig. 8 . It consists of four operating modes set by the four push buttons SW1, SW2, SW3, and SW4 as shown in Fig. 9. Generally, a calibration procedure should be carried out before all measurements are conducted in order to eliminate the electromagnetic interference by the magnetic fields from the machinery itself and from external sources. In the beginning of calibration procedure, the calibration button SW4 is pressed. Then, the OBFAM is rotated for two complete circles. Finally, the button SW4 is pressed again to finish the calibration procedure.

After calibration, the measuring procedure can be started. There are two measuring modes: normal mode with button SW1 and continuous mode with button SW2. The measured data can be shown on an LCD display board. The normal mode is suitable for measuring a field with a regular shape such as rectangles or polygons. Its procedure is to press button SW1 at every point of turn when the OBFAM goes along the boundary of the test area and returns to the origin. The continuous mode is specially designed for irregular shapes. Its operation is simply to press button SW2 at the starting point and when the OBFAM returns to the origin respectively. Furthermore, the function of button SW3 is to display the instantaneous azimuth angle during measuring.

This prototype utilizes W78E516B-40 microprocessor made by Winbond CO., LTD for calculation. As the pins from P14 to P17 receive signals from the four push buttons SW1, SW2, SW3, and SW4 which specify the desired mode, a proximity switch mounted on the wheel shaft of the vehicle sends a square-wave signal to the microprocessor serving as an interrupt vector INT0. The number of ON/OFF cycles for 


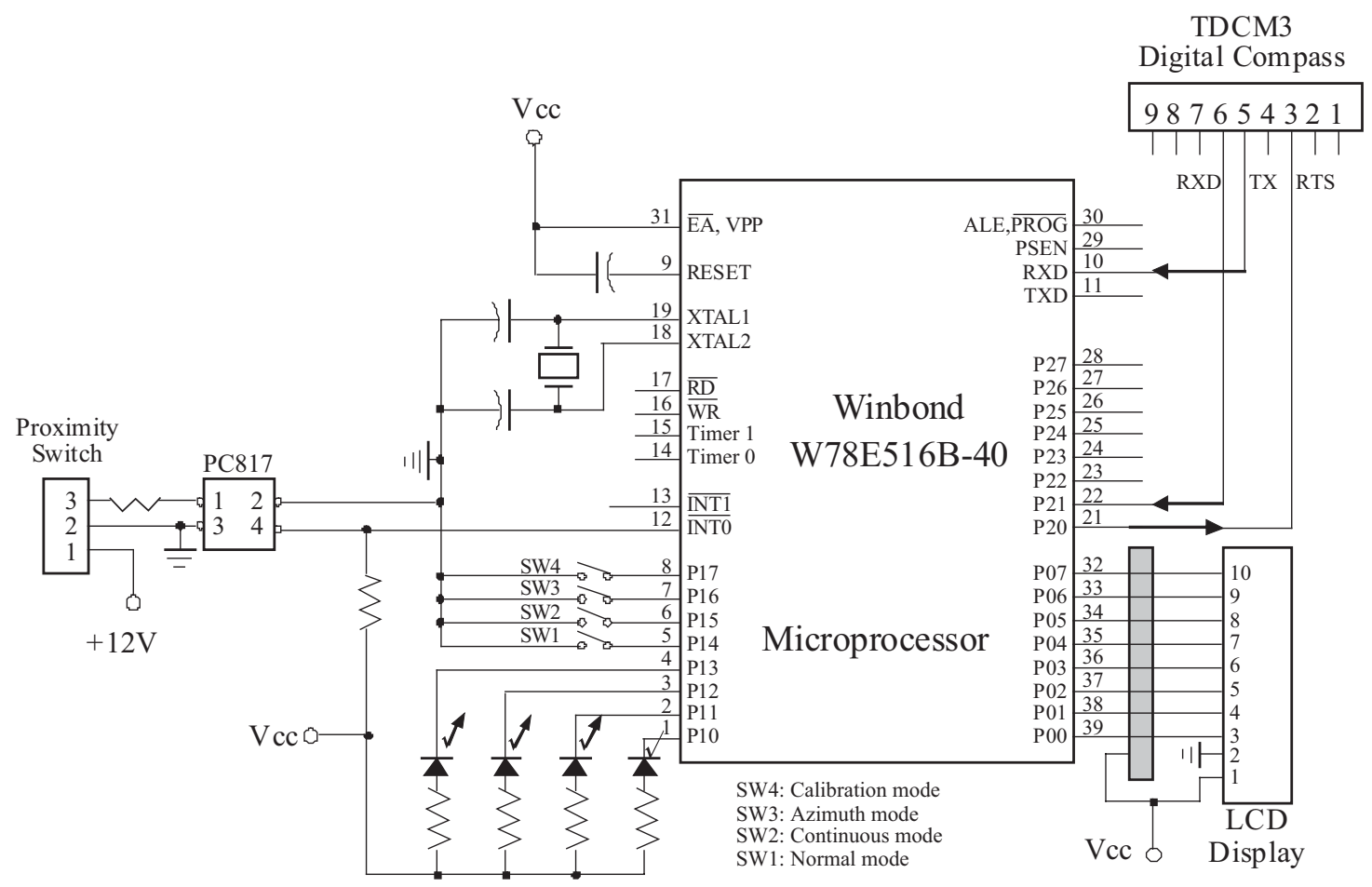

Fig. 9. Block diagram of the OBFAM circuit.

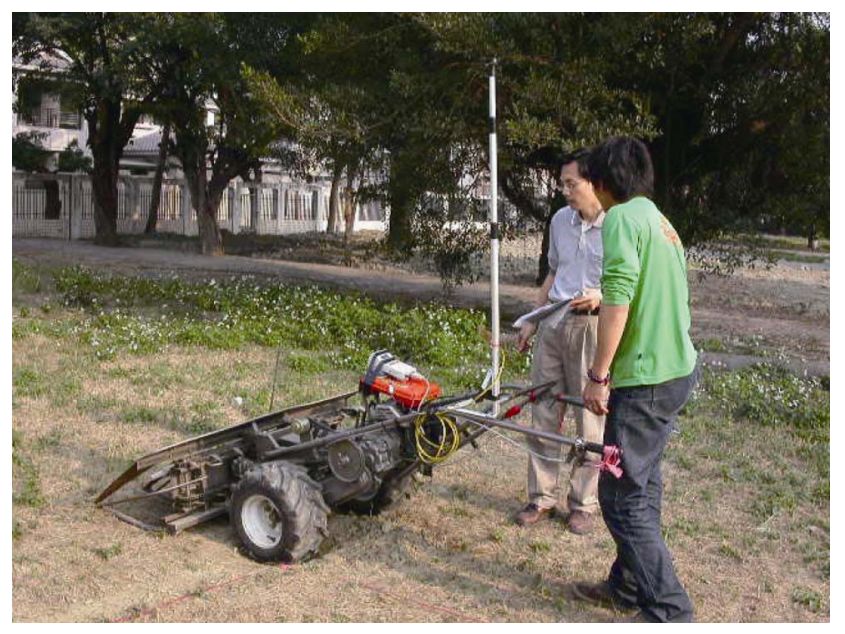

Fig. 10. Photograph showing testing of the OBFAM prototype.

the square-wave signal is counted by the microprocessor to get the number of rotations for the wheel shaft as well as the corresponding moving distance of the vehicle. Meanwhile, the microprocessor pin P20 sends a signal to pin 3 (RTS) of the digital compass for requesting required data. The digital compass will then send back three byte data Status, $\theta_{\mathrm{MSB}}$ and $\theta_{\mathrm{LSB}}$ from pin 5 (TX) of the digital compass to pin 10 (RXD) of the microprocessor. The program embedded in the microprocessor reads these data to calculate the azimuth angle $\theta, \theta=\left(\theta_{\mathrm{MSB}}\right.$ $\left.\times 256+\theta_{\mathrm{LSB}}\right) / 2$ and the related area based on (8)-(10). The calculated value of area can be displayed on the LCD via
Table 1. Test results of the OBFAM prototype.

\begin{tabular}{|c|c|c|c|c|c|c|}
\hline NO & \multicolumn{3}{|c|}{ a corn harvester } & \multicolumn{3}{c|}{ a peanut gathering machine } \\
\hline & Mode & $\begin{array}{c}\text { Area } \\
\left(\mathrm{m}^{2}\right)\end{array}$ & $\begin{array}{c}\text { Error } \\
(\%)\end{array}$ & Mode & $\begin{array}{c}\text { Area } \\
\left(\mathrm{m}^{2}\right)\end{array}$ & $\begin{array}{c}\text { Error } \\
(\%)\end{array}$ \\
\hline 1 & Manual & 379 & 1.07 & Manual & 893 & 0.78 \\
\hline 2 & Manual & 371 & 0.80 & Manual & 888 & 1.33 \\
\hline 3 & Manual & 379 & 1.07 & Manual & 905 & 0.56 \\
\hline 4 & Manual & 379 & 1.07 & Manual & 912 & 1.33 \\
\hline 5 & Manual & 375 & 0 & Manual & 897 & 0.33 \\
\hline 6 & Auto & 380 & 1.33 & Auto & 919 & 2.11 \\
\hline 7 & Auto & 383 & 2.13 & Auto & 890 & 1.11 \\
\hline 8 & Auto & 377 & 0.53 & Auto & 920 & 2.22 \\
\hline 9 & Auto & 377 & 0.53 & Auto & 904 & 0.44 \\
\hline 10 & \multicolumn{7}{|c|}{ Auto } & 379 & 1.07 & Auto & 885 & 1.66 \\
\hline \multicolumn{6}{|c|}{$\begin{array}{c}\text { The accurate area to be } \\
\text { measured }=375 \mathrm{~m}^{2}\end{array}$} & \multicolumn{2}{c|}{$\begin{array}{c}\text { The accurate area to be } \\
\text { measured }=900 \mathrm{~m}^{2}\end{array}$} \\
\hline
\end{tabular}

output ports $\mathrm{P} 00 \sim \mathrm{P} 07$ of the microprocessor.

\section{TEST RESULTS OF THE SYSTEM}

The prototype of the OBFAM shown in Fig. 8 was installed in a vehicle for agricultural application and was tested on a selected field as shown in Fig. 10. The test results for manual mode and auto mode in Table 1 reveal that the errors of the measured values are sufficiently small. The feasibility of the developed OBFAM prototype is thus confirmed. 


\section{DISCUSSION AND CONCLUSION}

A prototype for an on-board field area meter (OBFAM) with an azimuth sensor and a distance sensor has been designed to provide measured area data for agricultural machinery such that the disagreement about the exact cultivated area can be resolved. It utilizes a simple compass for measuring azimuth angle and a proximity switch for measuring distance. Furthermore, It consists of a W78E516 microprocessor of 8051 series chip to perform the calculation required for calibration and contour integral. Real time values of azimuth angle and area can be obtained by the proposed OBFAM.

Tests carried out by installing the prototype on a peanut gathering machine and a corn harvester show that the errors of the measured data with these two modes are all less than $2.5 \%$. Furthermore, the total cost for the required components are less than 100 US dollars. Hence, due to its structural simplicity, easy installation, low manufacturing cost, high reliability, high accuracy, and excellent feasibility, the OBFAM is expected to offer great contribution to agricultural automation.

\section{ACKNOWLEDGMENTS}

The authors are indebted to Lee-Guan Factory and YuanChen Co., Ltd. for providing a corn harvester and a peanut gathering machine for testing. Furthermore, the authors would like to thank for the financial support from Ministry of Economic Affairs (CIUP project NO. 92U0082) in Taiwan and the assistance of professor Lee Bean-Yin.

\section{REFERENCE}

1. Hague, T., Marchant, J. A., and Tillett, N. D., "Ground based sensing systems for autonomous agricultural vehicles," Computers and Electronics in Agriculture, Vol. 25, pp. 11-28 (2000).

2. Primdahl, F., "The fluxgate magnetometer," Journal of Physics E: Scientific Instruments, Vol. 12, pp. 241-253 (1979).

3. Reitz, P. and Kutzbach, H. D., "Investigations on a particular yield mapping system for combine harvesters," Computers and Electronics in Agriculture, Vol. 14, pp. 137-150 (1996).

4. Ripka, P., "Review of fluxgate sensor," Sensors and Actuators A: Physical, Vol. 33, pp. 129-141 (1992).

5. Speckmann, H., "Providing measured position data for agricultural machinery," Computers and Electronics in Agriculture, Vol. 25, pp. 87-106 (2000). 